\title{
Aprovechamiento de semillas de Dipteryx odorata (Aublet.) Willd. (Shihuahuaco) como producto alimenticio
}

\author{
Dipteryx odorata (Aublet.) Willd. (Shihuahuaco) seeds \\ as a food product
}

\author{
Aparicio Limache Alonzo' \\ Universidad Nacional de Ucayali \\ apaforestal2011@hotmail.com
}

\section{RESUMEN}

Considerando la necesidad de contribuir a la diversificación de insumos alimenticios mediante el aprovechamiento masivo de las partes no maderables de las especies forestales tropicales con énfasis en la región Ucayali - Perú, se exploró la posibilidad de utilizar a las semillas o almendras del Dipteryx odorata como producto alimenticio de consumo humano. Para ello, se sometió a un análisis nutricional, determinación de contenido de minerales y se complementó con una investigación bibliográfica para identificar los antecedentes de uso y exigencias que implica su conversión en un bionegocio. Así, se determinaron la materia seca, extracto etéreo, fibra cruda y proteína cruda en $88,89 \%, 19 \%, 16,75 \%$ y $1,51 \%$ respectivamente. Al cuantificar los oligoelementos como el zinc, hierro, cobre, sodio, plomo, magnesio se encontraron valores de 39,$29 ; 25,86 ; 12,36 ; 4,79 ; 3,04 ; 1,31$ ppm respectivamente. La investigación bibliográfica, demostró que en áreas rurales dichas almendras son utilizadas en la dieta alimenticia de la población. En Europa, son consideradas gourmet básico para la coctelería e ideal para repostería exótica. Se concluye indicando que por su contenido nutricional y de minerales las almendras de shihuahuaco en cantidades pequeñas, son aptas para consumo humano. Como limitante para su aprovechamiento en bionegocio, es el contenido de plomo que requiere desarrollo de estudios previos para neutralizar su efecto nocivo que genera problemas gastrointestinales por exceso de consumo.

Palabras clave: Nutrientes, minerales, semilla de Dipteryx odorata.

\begin{abstract}
Considering the need to extend the range of benefits of non-timber forest products from tropical species, especially, from the Ucayali region in Peru; this study aimed to explore the possibility of using Dipteryx odorata seeds as a food product for human consumption. Shihuahuaco seeds were subjected to nutritional and mineral content analysis, and research on the seeds' utilization, and on its requirements to transform the product into a "biobusiness", were performed. Thus, dry matter, ether extract, crude fiber and crude protein contents were $88,89 \%$, $19 \%, 16,75 \%$ and $1,51 \%$ respectively. The trace elements quantification such as zinc, iron, copper, sodium, lead, magnesium reported values of 39,29 ; 25,$86 ; 12,36 ; 4,79 ; 3,04 ; 1,31$ ppm respectively. The literature search showed that in rural areas these seeds are used in the population diet. And in Europe, they are considered gourmet food ideal for exotic pastries and cocktails. It has been concluded that nutritional and mineral content of the shihuahuaco seeds make it suitable for human consumption in small quantities. The lead content is a limiting factor for the seeds' utilization as a "biobusiness", requiring further studies to neutralize its harmful effects for excess consumption which generates gastrointestinal problems.
\end{abstract}

Keywords: Nutrients, minerals, Dipteryx odorata seeds.

Historial del artículo:

Recibido: 3 de noviembre de 2015. Aprobado: 11 de diciembre de 2015. Disponible en línea: 30 de diciembre de 2015

1 Doctor en Ciencias Ambientales y Desarrollo Sostenible, docente asociado de la Universidad Nacional de Ucayali. 


\section{INTRODUCCIÓN}

Las especies forestales de los bosques tropicales como el Dipteryx odorata (Aublet.) Willd. (Shihuahuaco) de la familia Fabaceae, entre otros se caracterizan por el contenido de una infinidad de compuestos bioquímicos apropiados para el aprovechamiento como insumo de productos alimenticios, industriales y medicinales tal como puntualiza Oliveros (1). Por experiencia personal, se sabe que en el área rural las almendras o semillas son utilizadas en la alimentación humana. Como tal representan un potencial económico desperdiciado por desconocimiento de su contenido de nutrientes y de minerales. Las características del fruto y de la semilla de Dipteryx odorata, se muestra en las figuras 1,2 y 3.

Algunos antecedentes se reporta en Brasil, donde Fernandes motivado por el uso de las almendras en la gastronomía de los pobladores de la zona, analizó la composición química y determinó valores de lípidos entre 397 - $437 \mathrm{~g} / \mathrm{kg}$, proteínas entre 238 - 281 g/kg, aminoácidos entre 83 - 103 \% (2). Indica que el almendro puede ser utilizado como fuente complementaria de proteínas y una excelente opción para dietas saludables. Fuchs confirma que en Europa la semilla del shihuahuaco (tonka), es considerado como esencia para la gastronomía, los países exportadores son Brasil y Venezuela (3).

Soares da Cruz caracterizó las propiedades moleculares de la semilla de shihuahuaco y encontró $244 \mathrm{~g} / \mathrm{kg}$ de proteína sobre una base de peso seco. Las albúminas y globulinas representaron 14 y 3,3\% en peso respectivamente. La proteína total de la harina y la globulina mostró valores de digestibilidad in vitro de $85,59 \%$ y $90,54 \%$ con relación a la caseína. Reporta predominio de una fracción de proteína tipo legumina (4).

En relación a estudios sobre la importancia de los nutrientes, se tiene a Bugarín-Montoya que creó una metodología para estimar la acumulación diaria de materia seca en la biomasa aérea del tomate durante el período de crecimiento (5). Pérez evaluó la actividad antibacteriana de extractos metanólicos, clorofórmicos y extractos etéreos de las hojas de dos especies infestado con bacterias. Así, determinó que los etéreos no son antibacterianos (6).

De los oligoelementos, Rodríguez estudió la deficiencia de zinc y hierro en la dieta de una población de 292 menores de 15 años. Como resultado, encontró bajo consumo de dichos elementos y presencia de inhibidores de absorción (7). Bisbe afirma que el hierro intravenoso resultó útil para la mejoría de los pacientes por lo que representa una nueva terapéutica a valorar en medicina transfusional (8).

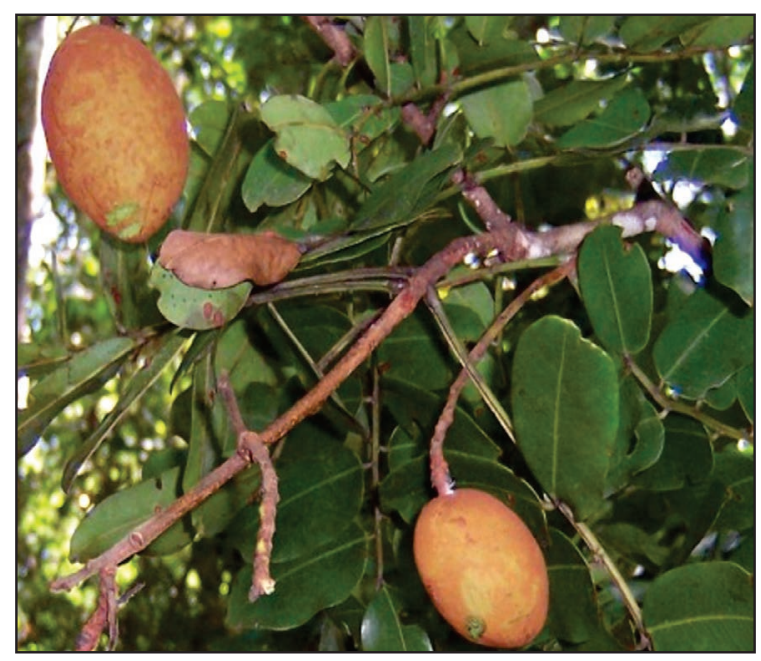

Figura $\mathrm{N}^{\circ} 1$ : Fruto.
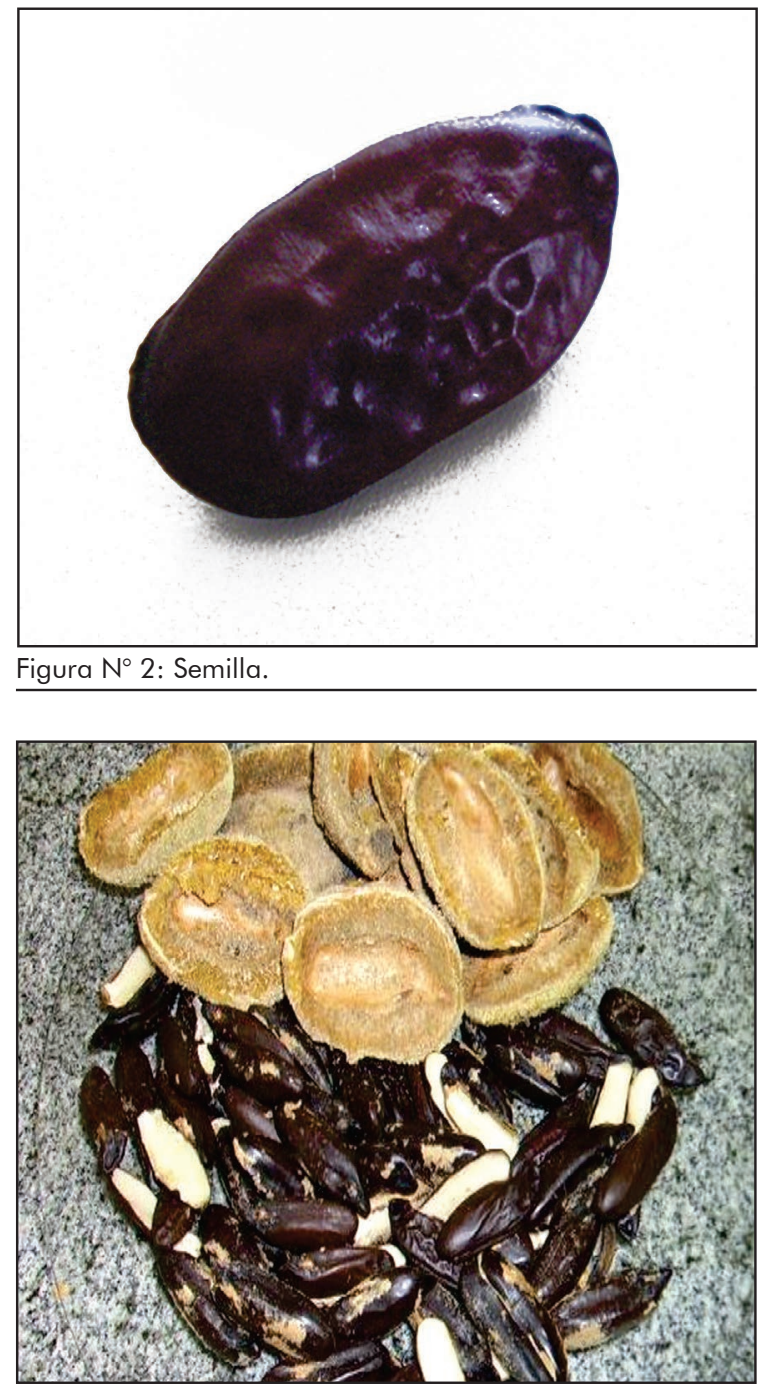

Figura $\mathrm{N}^{\circ}$ 3: Semilla liberada del fruto. 
De los gajos y corteza de la especie Parahancornia amapa (amapazeiro) y Apocynaceae, Henrique extrajo diclorometánico, $\beta$-sitosterol, estigmasterol, além de triterpenóides pentacíclicos, $\alpha$-amirina, $\beta$-amirina, lupeol e friedelina. Del extracto metanólico de la corteza aisló el alcaloide indólico isositsiriquina (9).

En este contexto, el objetivo consistió en analizar la posibilidad de crear un bionegocio de productos alimenticios de consumo humano basado en el contenido de nutrientes y de oligoelementos de las semillas del shihuahuaco.

\section{MATERIAL Y MÉTODOS}

Las semillas se recolectaron de árboles de regeneración natural localizada en el Centro de Investigación y Capacitación Forestal (CICFOR - Macuya). En el mes de agosto se realizaron el análisis químico proximal de componentes nutricionales. Dicho trabajo fue efectuado en el Laboratorio de Productos Forestales No Maderables de la Universidad Nacional de Ucayali de la ciudad de Pucallpa.

Entre los meses de septiembre y octubre se determinó el contenido de minerales. Este trabajo se ejecutó con un Espectrofotómetro de Absorción Atómica en el Laboratorio de Química de la Universidad Nacional del Centro del Perú con sede en Huancayo.

El método utilizado fue la identificación, contrastación de la información. Se identificaron los elementos nutricionales más representativos y el contenido de minerales. En seguida, mediante una investigación bibliográfica, se contrastaron las funciones que cumplen cada componente en el organismo del ser humano. Finalmente, basado en los resultados, se hizo una propuesta de su utilización como insumo para consumo alimenticio.

La investigación fue básica de tipo exploratoria no experimental, como tal no se trató de demostrar una hipótesis. Tampoco se adaptó a un Diseño Estadístico definido. Para garantizar la calidad de la información, los trabajos en Laboratorio se realizaron con tres repeticiones, de modo que los datos validados fueron los promedios.

El procedimiento consistió en seleccionar tres árboles con frutos diseminados al suelo, como tal, la recolección se hizo del suelo. Los frutos se trasladaron al laboratorio. En ésta, cada intervención fue efectuada a través de protocolos convencionales. Basado en los resultados se buscó información bibliográfica para determinar su importancia.

\section{RESULTADOS}

\section{Componentes nutricionales de la semilla de Dipteryx odorata (Aublet.) Willd.}

Como resultado del análisis químico proximal, la semilla del shihuahuaco registró alta concentración de materia seca $(88,89 \%)$, el extracto etéreo (19\%) y fibra cruda $(16,75 \%)$ alcanzaron concentración media y la proteína cruda $(1,51 \%)$ considerado como baja.

\section{Contenido de oligoelementos}

En la tabla 2, se aprecia los minerales representativos de la semilla del Dipteryx odorata al Zinc, Fierro y cobre con $0,39,0,26$ y $0,12 \mathrm{~g}$ respectivamente. Como elementos potenciales se visualiza al sodio, plomo y Magnesio con 0,05, 0,03 y 0,01 g respectivamente. El plomo se presenta como un elemento no apto para

Tabla $N^{\circ} 1$ : Componentes nutricionales de la semilla Dipteryx odorata (Datos expresados en \%).

\begin{tabular}{lcccc}
\hline Muestras & $\begin{array}{c}\text { Materia } \\
\text { seca }\end{array}$ & $\begin{array}{c}\text { Extracto } \\
\text { etéreo }\end{array}$ & $\begin{array}{c}\text { Fibra } \\
\text { cruda }\end{array}$ & $\begin{array}{c}\text { Proteína } \\
\text { cruda }\end{array}$ \\
\hline I & 90,20 & 19,51 & 16,67 & 0,94 \\
II & 88,24 & 18,75 & 16,83 & 1,79 \\
III & 88,24 & 18,75 & 16,76 & 1,79 \\
Suma & 266,67 & 57,01 & 50,26 & 4,52 \\
Prom & 88,89 & 19,00 & 16,75 & 1,51 \\
\hline
\end{tabular}

Tabla $N^{\circ}$ 2: Oligoelementos de la semilla de Dipteryx odorata (Datos expresados ppm).

\begin{tabular}{lrrrrrr}
\hline \multicolumn{1}{c}{ C } & Zinc & Hierro & Cobre & Sodio & Plomo & Magnesio \\
\hline I & 39,81 & 27,01 & 12,38 & 4,81 & 2,94 & 1,72 \\
II & 39,09 & 25,35 & 12,35 & 4,77 & 3,11 & 1,13 \\
III & 38,99 & 25,23 & 12,35 & 4,81 & 3,07 & 1,06 \\
Suma & 117,88 & 77,59 & 37,07 & 14,38 & 9,11 & 3,92 \\
Prom & 39,29 & 25,86 & 12,36 & 4,79 & 3,04 & 1,31 \\
\hline
\end{tabular}


consumo humano.

\section{Resultados de la investigación bibliográfica}

Al contrastar la relación de los componentes nutricionales de las semillas del shihuahuaco, con la información bibliográfica, se encontró antecedentes sorprendentes. Cada componente nutricional cumple una actividad especializada en el mantenimiento de la calidad de vida de los seres. En efecto, la presencia o ausencia de los nutrientes en el organismo está asociada con la vida saludable del individuo

Del mismo modo, los oligoelementos están mucho más relacionados con la salud. La deficiencia y exceso de estos elementos implica enfermedades que conduce a la muerte del individuo. El hierro, el zinc y el cobre, actúan en alianza estratégica para regular el sistema completas y determinó el peso seco. Como resultado, notó que la planta acumuló 1003,4 g de materia seca, destinando $54 \%$ hacia los frutos y $46 \%$ hacia las hojas y tallos (10).

Rojas-Hernández, también demostró acumulación de materia seca en cuatro cultivares de Brachiaria, con base en su comportamiento agronómico a diferentes edades de corte. Como resultado obtuvo una

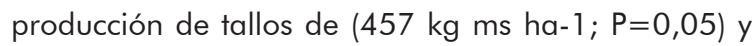
materia seca total $(1,125 \mathrm{~ms} \mathrm{ha-} 1 ; \mathrm{P}=0,05)$. Concluye que los cultivares con mejor desempeño agronómico fueron Brachiaria ruziziensis $X \quad B$. brizantha, sin diferencia en la producción de materia seca entre cultivares (11),

Por otro lado, el porcentaje de extracto etéreo del shihuahuaco fue $19 \%$, ésta cantidad está catalogado

Tabla № 3: Resumen de la función de nutrientes y oligoelementos en el organismo.

\begin{tabular}{ll}
\hline \multicolumn{1}{c}{ Elementos } & \multicolumn{1}{c}{ Funciones } \\
\hline $\begin{array}{l}\text { Nutrientes } \\
\text { Materia seca } \\
\text { extracto }\end{array}$ & Acumulación de materia seca durante el ciclo de crecimiento. \\
$\begin{array}{l}\text { Fibra cruda } \\
\text { Proteína bruta }\end{array}$ & $\begin{array}{l}\text { Viabiliza intercambio de cationes o sustancias en el organismo. } \\
\text { Interviene en la digestibilidad de los alimentos consumidos. }\end{array}$ \\
\hline & \\
Tabla No 4: Algunas funciones que cumplen los oligoelementos en el organismo. \\
\hline Oligoelementos
\end{tabular}

circulatorio de los individuos.

\section{DISCUSIÓN}

\section{Contribución de los nutrientes del shihuahuaco a dietas saludables}

En la tabla 1, se demuestra que el porcentaje de materia seca del shihuahuaco es $88,98 \%$ catalogado como relativamente alto. Según Núñez, la materia seca cumple función de absorción, acumulación e intercambio o distribución de sustancias. Para demostrar su afirmación, analizó el ciclo de crecimiento del tomate midiendo semanalmente altura, número de hojas y el área foliar de la hoja madura. Cada tres semanas hizo muestreos de biomasa a plantas como medio. En este estudio, no se determinó las sustancias que conforman dicho extracto etéreo, pero se supone que son numerosos. Por ejemplo, Abreu al estudiar el extracto etéreo de frutos de Bromelia pinguin L. (piña de ratón) mediante el sistema acoplado, detectó la presencia de los ácidos 2-pentenodioico, octanodioico, ftálico, cítrico, nonanodioico, 12-metiltetradecanoico, palmítico, oleico, linolénico, esteárico, 11, 14, 17-eicosatrienoico y 11, 14-eicosadienoico (12).

Bressani y Arroyave analizaron la humedad, nitrógeno, extracto etéreo, fibra cruda, hierro y fósforo, de 23 tipos de maíz de origen guatemalteco, mexicano y venezolano ajustando valores a una humedad promedio de $10 \%$. El promedio de nitrógeno fue de $1,52 \%$ (s 0,22$)$, el de extracto etéreo, $5,63 \%(\mathrm{~s} 0,97)$, el de fibra cruda, 1,40\% (s 0,21), el de hierro $3,00 \mathrm{mg}$ 
por $100 \mathrm{gm}(\mathrm{s.}$ 0,92) y el de fósforo, $320 \mathrm{mg}$ por $100 \mathrm{~g}$ (s 58). Se determinó que el ambiente y la genética son factores que influyen en el valor nutritivo y cantidad de elementos especialmente en cuanto al nitrógeno y al extracto etéreo (13).

También el porcentaje de fibra cruda del shihuahuaco se cataloga como medio porque según la tabla 1 , llega a $16,75 \%$. Al igual que el extracto etéreo cumple una función importante en el organismo. Así, en un estudio sobre fracciones del tamaño de partícula de la fibra del musgo Sphagnum magellanicun, $\mathrm{T} 1(1,4$ $\mathrm{mm}) ;$ T2 $(1,0 \mathrm{~mm}) ;$ T3 $(0,425 \mathrm{~mm}) ;$ T4 $(0,180 \mathrm{~mm})$. Villarroel obtuvo mejores respuestas de capacidad de absorción, retención de agua, absorción de moléculas orgánicas con el T3, con excepción de la capacidad de intercambio catiónico cuyo mejor resultado se presentó en T1 (14).

Canché-Escamilla, estudió la factibilidad de obtención de celulosa a partir de residuos agrícolas del banano (pseudo tallo y pinzote). El proceso consistió en cuatro etapas: hidrólisis ácida, cloración, extracción alcalina y blanqueo. Se obtuvo pesos moleculares de 90,000 y de 49,000 cuando se usó un $\mathrm{pH}$ de 9,2 y 8,4 en la etapa de cloración, respectivamente; se obtuvieron mejores rendimientos con las fibras de pinzote (15).

El porcentaje de proteína cruda del shihuahuaco, apenas llega a 1,51\%. Se sabe que este elemento es esencial para la formación de aminoácidos, coadyuva la digestibilidad y el metabolismo. Cabe indicar que el contenido de proteína bruta (PB) de los vegetales refleja su calidad como alimento saludable y ésta varía en alto grado de una especie a otra. En el caso específico del shihuahuaco, al notar escasa presencia se deduce que cumple una función limitada, por ende su calidad alimenticia también es limitada.

Al respecto, Cruz evaluó el efecto de 4 niveles de la relación proteína cruda / energía bruta $(P / E=50,60$, 70 ○ $80 \mathrm{mg}$ prot. $/ \mathrm{kcal}$, para niveles de proteína de $=$ $20,25,30 \circ 35 \%)$ y dos proporciones de proteína vegetal (soya y trigo)/ animal (pescado y camarón). Las dietas se evaluaron en camarones $L$. vannamei y $L$. stylirostris, en dos bioensayos de crecimiento de 28 día. Comprobaron que la digestibilidad de la proteína fue ligeramente inferior en L. vannamei comparado con L. stylirostris (88 vs $90 \%$ ), mientras la digestibilidad de materia seca fue mayor en L. vannamei que en $\mathrm{L}$. stylirostris (70 - $79 \%$ vs 69 - $75 \%$ ) (16).

Chirino encontró variación del contenido de proteína bruta de especies forrajeras comparadas entre un pastizal de verano con dominancia de Digitaria califórnica, otro de invierno como Poa ligularis y Piptochaetium napostaense; el tercero con predominio de Pappophorum caespitosum en verano y con Medicago minima y Erodium cicutarium en invierno.
En la primera especie de verano obtuvo $14,4 \%$ de proteína bruta (PB), en el segundo pastizal los valores fueron $8,5 \%$ y $12,4 \%$ de $\mathrm{PB}$, respectivamente, en el tercero encontró valores de $9,8 \%$ y $23,8 \%$ de $\mathrm{PB}$, respectivamente (17).

\section{Función de los oligoelementos del shihuahuaco en las dietas saludables}

En la tabla 2, se aprecia como minerales representativos de la semilla del Dipteryx odorata al zinc, hierro y cobre. Como elementos potenciales se visualiza al sodio, plomo y magnesio. Con excepción del plomo, los elementos citados son claves de las dietas saludables por ende en la salud de los comensales.

El zinc del shihuahuaco, según tabla 2 es 39,29 ppm, es el oligoelemento más representativo de mucho valor para la salud. En efecto, Rubio, afirma que el zinc tiene función cardio respiratoria y promoción de fuerza en personas sanas y en atletas, también interviene en la regulación de la presión sanguínea, actuando por lo tanto en ciertos tipos de hipertensión arterial. Una terapia con zinc aumenta la recuperación de pacientes que sufren anorexia nerviosa por incrementar la ganancia de peso y reducir la ansiedad y la depresión (18).

El hierro es otra contribución esencial de las semillas del shihuahuaco para la dieta saludable. Con los 25,86 ppm de concentración, se presenta inclusive como alternativa de una dieta medicinal cuya aplicación sería preventiva. Al respecto, Ortega, Boccio, y otros indican que el hierro es el componente de la hemoglobina que distribuye el oxígeno a las células y evacua el dióxido de carbono. Se encuentra formando dos grupos, uno es hémico y el otro no hémico, el hierro de tipo hémico, es el que forma parte de la hemoglobina, mioglobina, citocromos y muchas otras hemoproteínas. En cambio el no hémico corresponde al hierro que está formado por sales inorgánicas que contiene los alimentos de origen vegetal y en los preparados farmacéuticos (19) (20). Para Cardero la fuente de hierro son los cereales, raíces, tubérculos, carnes de aves, pescado, ácido ascórbico entre otros (21).

Al evaluar prevalencia de anemia en niños, de mujeres fértiles y gestantes, González encontró $26 \%$ de prevalencia de anemia en niños de 1 a 6 años. El 56,2 $\%$ de los niños presentó algún grado de deficiencia de hierro (ferritina menor de $24 \mathrm{ng} / \mathrm{dl}$ ). La prevalencia de anemia en la mujer en edad fértil fue de $18,9 \%$ y en mujeres gestantes de $27,9 \%$. Sin embargo, según la definición de la OMS, un 58,5\% de las gestantes mostró algún grado de deficiencia (ferritina menor a $24 \mathrm{ng} / \mathrm{dl}$ ) (1). Recomienda fortalecer la educación nutricional a la población, para consumir alimentos con fuentes de hierro (22). 
El cobre es otro elemento importante para el organismo. El shihuahuaco según la tabla 2 contiene $12,36 \mathrm{ppm}$ de $\mathrm{Cu}$, valor relativamente alto por los múltiples aportes que brinda a una dieta saludable. En efecto, el cobre al igual que el hierro y el zinc, están presentes en todos los tejidos del cuerpo en especial en la sangre. Las fuentes de cobre son las ostras, los granos enteros, las legumbres, las nueces, las papas, vísceras, verduras de hoja oscura, las frutas deshidratadas, pimienta negra y otros. La deficiencia de cobre provoca osteoporosis y anemia. El exceso produce hepatitis, problemas renales, trastornos cerebrales y otros.

A fin de contar con información sobre la nutrición mineral de los bovinos para carne, la Asociación Americana de Dietética, extrajo 31 muestras de suelos, 311 de forrajes, 218 de sangre, 208 biopsias de hígado y 69 de pelo, clasificó las muestras de suelo como provenientes de dos tipos de campo "alto" y "bajo" y las de origen animal en vacas en lactancia y animales en crecimiento. Concluye que la deficiencia primaria de $\mathrm{Cu}$ observada corresponde a menos de 3 ppm, agravada en algunas áreas por elevados niveles de Mo y/o S (23).

También en el shihuahuaco se encontró sodio en 4,79 ppm y magnesio en 1,31 ppm. Las cantidades son aparentemente pequeñas pero la bibliografía consultada demuestra funciones de vital importancia para el organismo. En los últimos años gracias al adelanto alcanzado con los instrumentos de última generación se descubre que el consumo de ambos elementos definitivamente contribuye a una vida saludable.

Al respecto, Martínez-Ferrer investigó las concentraciones de $\mathrm{Ca} 2+, \mathrm{Mg} 2+$ y $\mathrm{Na}+$ en las aguas de consumo público y comparó con los objetivos nutricionales de estos minerales. Afirma que el agua contiene minerales, como el magnesio $(\mathrm{mg} 2+)$ y el sodio $(\mathrm{Na}+)$, con efectos potenciales para la salud. El aumento de $\mathrm{Na}+$ en la ingesta contribuye al desarrollo de hipertensión, mientras que el aumento del mg2 + se ha relacionado con una disminución de muerte súbita. Se deduce que el tipo de agua que se consume, es crucial para conservar la salud (24).

Por ejemplo, Alday da a conocer que el magnesio es empleado como fármaco con distintas indicaciones en reanimación, obstetricia, cardiología, cirugía cardíaca, tratamiento del dolor, anestesia, neumología y otros.

Finalmente el papel del magnesio en el organismo y sus propiedades farmacológicas siguen siendo objeto de estudio. Afirma que el conocimiento de sus características farmacológicas, clínicas y fisiológicas del magnesio, se ha vuelto imprescindible para el médico anestesiólogo (25).
Villegas estudió el efecto del magnesio en deportistas calificados. Se informó de un aumento de magnesio en el músculo durante el ejercicio prolongado, paralelo a su disminución en plasma, dicha disminución podría provocar convulsiones de tipo epiléptico en corredores; de hecho hubo un caso en el que fue el causante de un espasmo carpo-pedal en una tenista de 24 años durante un largo y disputado partido. Sin embargo, dosis altas de magnesio no han podido ser relacionadas con un aumento del rendimiento deportivo (26).

El plomo de las semillas del shihuahuaco, según la tabla 2 es 3,04 y según la tabla 4 tiene un efecto nocivo para la salud. Produce alteraciones gastrointestinales representadas por cólicos, diarrea, vómitos y afines. Así el plomo se visualiza como un elemento que limitaría el uso extensivo de dichas semillas en dietas saludables.

Al respecto, Ferrís afirma que la exposición crónica ocasiona alteraciones hematológicas, renales, cardiovasculares, retarda el crecimiento infantil y altera la presión sanguínea. Afecta la función reproductiva por la toxicidad testicular y de vesículas seminales, incrementa el riesgo de abortos y los lactantes presentan retraso físico y mental (27).

\section{Análisis del contenido de nutrientes}

Si se analiza la tabla 1 , se verifica que las semillas del shihuahuaco en orden de importancia, concentra alta cantidad de materia seca que llega hasta 88,89 $\%$, media de extracto etéreo $19,00 \%$ y fibra cruda de $16,75 \%$, baja de proteína bruta que apenas llega a $1,5 \%$. Al respecto, Canché-Escamilla, Villarroel y otros confirman que los nutrientes están conformados por moléculas que absorben e intercambia diversidad de sustancias útiles para el organismo $(15,14)$.

Los trabajos de Abreu; Bressani y Arroyave; y Pérez confirman que los nutrientes contienen numerosos compuestos bioquímicos que coadyuvan los enlaces bioquímicos $(12,13,6)$. Analizando, los trabajos reportados por Rojas-Hernández, Bugarín-Montoya y Núñez-Ramírez, se nota que los nutrientes acumulan biomasa especialmente en la etapa de crecimiento $(11,5,10)$. La proteína bruta es un compuesto importante para una dieta saludable pero en la semilla del shihuahuaco no supero el 1,51 \%, por tanto es insignificante.

Estos antecedentes, hace pensar que la semilla de shihuahuaco es una alternativa para utilizar como insumo de una dieta saludable para humanos. Mayor provecho se obtendría destinando a la dieta de niños en edad escolar. Ellos necesitan mayor consumo de nutrientes por encontrarse en pleno desarrollo. 


\section{Análisis del contenido deoligoelementos}

En la tabla 2, se aprecia como minerales representativos de la semilla del Dipteryx odorata al zinc, hierro, cobre sodio, plomo y magnesio. Estos en conjunto regulan la presión sanguínea, reduce la ansiedad y la depresión de los pacientes. Por ejemplo Rubio comprobó que la prevalencia de anemia en niños presentó algún grado de deficiencia de hierro (18).

El cobre interviene en solución de problemas relacionados con el hueso. Cuando hay deficiencia provoca osteoporosis y anemia. Cuando hay exceso produce hepatitis, problemas renales, trastornos cerebrales y otros. Para Martínez-Ferrer el exceso de ingesta de sodio contribuye al desarrollo de hipertensión, mientras que el aumento del $\mathrm{Mg} 2+$ se ha relacionado con una disminución de muerte súbita (24).

Alday afirma que el magnesio es un fármaco útil para la cardiología, cirugía cardíaca, tratamiento del dolor, anestesia, neumología y otros (25). Villegas afirma que el aumento de magnesio en el organismo provoca convulsiones de tipo epiléptico (26). Todos los autores consultados coinciden en señalar que la deficiencia y el consumo en exceso siempre generan alteraciones.

Estos antecedentes confirman que el consumo de las semillas de shihuahuaco, constituye una alternativa de primer orden para consumo humano. Pues, se sabe que el consumo de oligoelementos tan importantes como el hierro, zinc y cobre, actuarán como preventivo para evitar enfermedades relacionadas con el sistema circulatorio.

Fuchs confirma que el haba tonka es la semilla del Dipteryx odorata, de la familia de las Fabaceae, originario del Orinoco. El nombre proviene de la lengua nativa de la Guayana francesa. Francia fue el primer país que importó estas semillas para utilizar como aromatizante del tabaco luego se convirtió en un ingrediente en repostería y cosmética. En 1868 se descubrió que contiene cumarina, un compuesto que en grandes dosis produce coagulación de la sangre. Por este motivo su uso alimentario está prohibido en EEUU, en cambio en Europa se consigue sin problemas en tiendas especializadas en especias (3).

Sin embargo, un pequeño problema es el plomo presente en las semillas del shihuahuaco, que en la tabla 2 es 3,04 ppm. Ésta en exceso produce alteraciones gastrointestinales. Por tanto, se convierte en una limitante para lanzar categóricamente a la citada semilla como insumo de dietas saludables especialmente en la Región Ucayali.

\section{Opción de industrialización de las semillas}

En resumen tanto por el contenido de nutrientes como por los oligoelementos presentes la alternativa de uso de las semillas del shihuahuaco como insumo alimenticio de humanos es una opción viable. Por el aspecto nutricional que refleja bajo contenido de proteína bruta su aporte al organismo no es contundente. Sin embargo por los oligoelementos como hierro, zinc y cobre, no cabe duda que es un buen insumo alimenticio, refuerza la propuesta de uso como alimento medicinal de tipo preventivo especialmente para personas de la tercera edad que son más propensos a enfermedades derivados de un sistema circulatorio deficiente.

Del valor comprobado de las semillas del shihuahuaco como alimento apto para dieta alimenticia, a la industrialización o conversión de ésta en un bionegocio, existe un tramo largo que recorrer. En las condiciones de la Región Ucayali - Perú, destacan dos limitantes. La primera es el contenido de plomo y el segundo es la escasa disponibilidad de semillas en el bosque, ésta última se agrava por la estacionalidad de la cosecha.

La solución del primer limitante, es posible mediante aplicación de experimentos de descarte. Es más, a la fecha se cuenta con tecnología sofisticada para superar este problema. En cambio el segundo limitante se relaciona con la naturaleza de la especie.

En efecto, su fructificación es irregular. Al respecto, Moçambite dice que varía entre 3 a 7 años. Por árbol se cosecha entre 10 a $15 \mathrm{~kg}$ de semilla/año. El shihuahuaco en la actualidad es objeto de una tala indiscriminada. Su madera es objeto de exportación por ende la demanda es inusual. Cada año, los árboles de shihuahuaco en el bosque se reducen dramáticamente entre otros por tala ilegal (28).

Si se opta por una plantación forestal de shihuahuaco, su fructificación inicia mínimo a los 20 años. A nivel Perú no se cuenta con plantaciones de shihuahuaco. Lo contrario ocurrió en Venezuela y Brasil donde sus respectivos Gobiernos adoptaron en la década de 70 una política de repoblamiento de shihuahuaco. Hoy cosechan cientos de $t$ de este producto y exportan a Europa como producto no tradicional, (3).

Por otro lado, estudios realizados en Bolivia por Gutiérrez y Silva demuestran que la densidad básica del shihuahuaco es $0,91 \mathrm{~g} / \mathrm{cm} 3$, la topa en cambio tiene $0.13 \mathrm{~g} / \mathrm{cm} 3$ (29). Por esta razón está catalogado como una especie de alta densidad. Según Limache, las especies de alta densidad básica, tienen alta capacidad de la captura de carbono (30).

Estas dos características visualizan la priorización 
de un programa de repoblamiento agresivo de esta especie especialmente con fines de reducción de huella de carbono. Si a ello se agrega la posibilidad de aprovechamiento de sus semillas, no cabe duda que el shihuahuaco es una especie de uso múltiple que

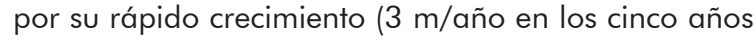
iniciales) podría generar riqueza inusitada.

\section{Aporte científico del estudio}

El aporte principal del estudio es redescubrir un producto nuevo para la alimentación y poner al servicio de la población por ahora de manera limitada. La propuesta se sustenta con una investigación de su contenido de nutrientes y oligoelementos. Si bien es cierto que existen antecedentes de uso, éstos se hicieron empíricamente.

Un sustento técnico complementario es la experiencia personal sobre consumo de las almendras de shihuahuaco por pobladores del área rural en la Región Ucayali, Perú. También se tiene el reporte de Fernandes, que pone en evidencia el uso de dichas almendras en la dieta alimenticia (2). Fuchs afirma que en Francia la semilla de shihuahuaco es un ingrediente gourmet básico para la coctelería y repostería exótica (3).

\section{Determinación del contenido nutricional y de minerales de las semillas}

Otro aporte del estudio es poner en evidencia el contenido de nutrientes para decidir el uso de las semillas. En efecto, sabiendo que tiene alto porcentaje de materia seca, medio de extracto etéreo y fibra cruda así como baja de proteína bruta, es posible decidir su uso. Esta constituye un fundamento para su futura industrialización.

De igual modo, sabiendo que tiene zinc, hierro y cobre en alta cantidad, ya es posible orientar el uso como alimento de personas de tercera edad que padecen de problemas en el sistema circulatorio. Sin embargo, queda establecido que no es posible industrializar las semillas sin antes controlar los efectos del plomo que es pequeña, pero dañina para la salud.

Las conclusiones respecto a este trabajo son:

Los oligoelementos de las semillas del shihuahuaco en orden de importancia son: Zinc, hierro, cobre, sodio, plomo y magnesio con $39,29,25,86,12,36,4,79$, 3,04, y 1,31 ppm respectivamente.

Las semillas del shihuahuaco registran $88,89 \%$ de materia seca, $19 \%$ de extracto etéreo, $16,75 \%$ de fibra cruda y $1,51 \%$ de proteína bruta.
La investigación bibliográfica demostró que tiene alto porcentaje de materia seca, media de extracto etéreo y fibra cruda pero baja en proteína. En estas condiciones su aporte a la salud es sólo para coadyuvar la absorción de moléculas orgánicas e intercambio de cationes.

Por la presencia en mayor grado del zinc, hierro y cobre, las semillas del shihuahuaco se presentan como una alternativa para insumo alimenticio de personas con problemas cardiovasculares en especial los adultos mayores.

El plomo aunque es 3,04 ppm representa un riesgo para la salud por sus antecedentes cancerígenas, antes de promover el consumo masivo de las semillas del shihuahuaco es recomendable desarrollar estudios de neutralización de este elemento.

La propuesta de uso como alimento para humanos se basa en antecedentes de uso en áreas rurales y en gastronomía de alta calidad utilizado en Europa y Estados Unidos.

El aporte principal del estudio es redescubrir un producto nuevo para la alimentación y poner al servicio de la población por su alto contenido de oligoelementos útiles para la salud.

\section{REFERENCIAS BIBLIOGRÁFICAS}

1. Oliveros A, Cordero I, Paredes D, Buendia D, Domínguez F. Extracción y cuantificación de cumarina mediante HPLC-UV en extractos hidroetanolico de semillas de Dipteryx odorata. Revista latinoamericana de química. 2011; 39(12): p. 17-31.

2. Fernandes D, Freitas J, Czedera L, Naves M. Nutritional composition and protein value of the baru (Dipteryx alata Vog.) almond from the Brazilian Savanna. J Sci Food Agric. 2010; 90(10): p. 1650 - 1655.

3. Fuchs L. Directo al paladar. [Online].; 2012 [cited 2015 Febrero 12. Available from: http://www. directoalpaladar.com/ingredientes-y-alimentos/ que-es-el-haba-tonka.

4. Da Cruz KS, Da Silva MA, de Freitas $O$, Neves VA. Partial characterization of proteins from baru (Dipteryx alata Vog) seeds. J Sci Food Agric. 2011; 91(11): p. 2006 - 2012.

5. Bugarín-Montoya R, Galvis-Spinola A, SánchezGarcía P, García-Paredes D. Acumulacion diaria de materia seca y de potasio en la biomasa aérea total de tomate. Terra. 2001; 20: p. 401 - 409.

6. Pérez $E$, Isaza G, Acosta M. Actividad antibacteriana de extractos de Phenax rugosus y Tabebuia chrysantha. Biosalud. 2007;(6): p. 59 - 
68.

7. Rodríguez D, Papale J, Dellan G, Torres $M$, et al. Deficiencia de Zinc y Cobre en menores de 15 años una poblacion rural de Venezuela. Boletín Médico de Postgrado. 2004; 20(2): p. 55 - 62.

8. Bisbe $E$, Rodríguez C, Ruiz A, Sáez $M$, et al. Uso preoperatorio de hierro endovenoso. Una nueva terapéutica en medicina transfusional. Rev. Esp. Anestesiol. Reanim. 2005; 52(9): p. 536 - 540.

9. Henrique, Nunomura R, Nunomura $S$, Silva $S$. Constituintes químicos isolados dos galhos e cascas de amapazeiro (Parahancornia amapa, Apocynaceae). Acta amazónica. 2014; 44(4): p. $533-538$.

10. Núñez-Ramírez F, Grijalva-Contreras RL, MacíasDuarte R, Robles-Contreras F, Ceceña-Duran C. Crecimiento, acumulacion y distribucion de materia seca en tomate de invernadero. Revista de Ciencias Biológicas y de la Salud. 2012; 14(3): p. 25 - 31.

11. Rojas-Hernández S, Olivares-Pérez J, JiménezGuillén R, Gutiérrez-Segura I, Avilés-Nova F. Producción de materia seca y componentes morfológicos de cuatro cultivares de Brachiaria en el Tropico. Avances de Investigacion Agropecuaria. $2011 ; 15(1)$ : p. 3 - 8.

12. Abreu J, Miranda M, Lora J. Extracto etéreo de frutos de Bromelia pinguin L. (piña de ratón) por el sistema acoplado CG-EM. Rev Cubana Farm. 2001; 35(1): p. 25 - 30.

13. Bressani R, Arroyave G, Scrimshaw N. EL Valor Nutritivo de las Variedades de maiz cultivadas en centro america: Nitrogeno, Extracto Etereo, Fibra Cruda y minerales. Panamá: Instituto de Nutrición de Centro América y Panamá ; 2001.

14. Villarroel M, Acevedo C, Yanez E, Biolley E. Propiedades funcionales de la fibra del musgo Sphagnum magellanicum y su utilización en la formulación de productos de panadería. Archivos Latinoamericanos de Nutrición. 2003; 53(4): p. 400-407.

15. Canché-Escamilla G, De los Santos-Hernández JM, Andrade-Canto S, Gómez-Cruz R. Obtención de Celulosa a Partir de los Desechos Agrícolas del Banano. Informacion Tecnologica. 2005; 16(1): p. $83-88$.

16. Cruz-Suárez E, Antimo-Pérez J, Luna-Mendoza N, Tapia-Salazar M, Guajardo-Barbosa C, et al. Relaciones proteína/energía y proteína vegetal/ animal optimas en alimentos de engorda para Litopenaeus vannamei y L. stylirostris. Mérida: Universidad autónoma de Nuevo León, Facultad de Ciencias Biológicas; 2000.

17. Chirino C, Norlader K, Robles M. Determinación de Proteina Bruta de algunas especies forrajeras de la Pampa. Revista de la Facultad de Agronomía. 1988; 3(2): p. 57 - 74.

18. Rubio C, González D, Martín-Izquierdo RE, Revert C, Rodríguez I, et al. El zinc: oligoelemento esencial. Nutrición Hospitalaria. 2007; 22(1): p. $101-107$.

19. Ortega M. Valor nutrimental de la pulpa fresca de aguacate Hass. In Actas V Congreso Mundial del Aguacate.; 2003; Michoacán: Secretaria de Desarrollo del Estado de Michoacán. p. 741 -748.

20. Boccio J, Salgueiro J, Lysionek A, Zubillaga $M$, Goldman $C$, et al. Metabolismo del hierro: conceptos actuales sobre un micronutriente esencial. ALAN. 2003; 53(2).

21. Cardero Y, Sarmiento R, Selva A. Importancia del consumo de hierro y vitamina $C$ para la prevención de anemia ferropénica. MEDISAN. 2009; 13(6).

22. González R. Biodisponibilidad del Hierro. Revista Costarrisence de Salud Pública. 2005; 14(26): p. $11-22$.

23. Asociación Americana de Dietética. Postura de la Asociación Americana de Dietética y de la Asociación de Dietistas de Canada: Dietas vegetarianas. J Am Diet Assoc. 2009; 109: p. $1266-1282$.

24. Martínez-Ferrer Á, Peris P, Reyes R, Guañabens N. Aporte de calcio, magnesio y sodio a través del agua embotellada y de las aguas de consumo público: implicaciones para la salud. Medicina Clínica. 2005; 131(17): p. 641 - 646.

25. Alday E, Uña R, Redondo FJ, Criado A. Magnesio en Anestesia y Reanimación. Rev. Esp. Anestesiol. Reanim. 2005; 52: p. 222 - 234.

26. Villegas JA, Zamora S. Necesidades nutricionales en deportistas. Archivos de Medicina del Deporte. $1991 ; 8(30)$ : p. 169 - 179.

27. Ferrís i J, García i J, López JA, García i F, Ortega $\mathrm{JA}$, et al. Enfermedades asociadas a la polución atmosférica por combustibles fósiles. Aspectos pediátricos. Rev Esp Pediatr. 2001; 57(3): p. 213 225.

28. Moçambite A, Morellato LP, Pereira A. Fenologia reprodutiva de Dipteryx odorata (Aubl.) Willd (Fabaceae) em duas áreas de floresta na Amazônia Central. Acta Amaz. 2008; 38(4): p. 643 - 650.

29. Gutiérrez V, Silva J. Información técnica para el procesamiento industrial de 134 especies maderables de Bolivia La Paz : FAO - PAFBOL; s/f.

30. Limache A. Método no destructivo recomendable para determinar carbono almacenado por fustales en boques tropicales de la Región Ucayali - Perú. Huancayo; 2014. 